\title{
Drugs for rare diseases
}

\author{
W. H. LYLE \\ M.D. \\ Dista Products Limited
}

\begin{abstract}
Summary
Pharmaceutical houses alone are equipped to market new medicines. The cost is very high, much of this arising from the preliminary toxicological and pharmacological work which is required in order to satisfy licensing authorities.

The introduction of a drug, even for a rare and lethal disease without such data, even if permitted by the authorities, would leave its manufacturer exposed to adverse publicity and litigation in the event of a mishap.

These considerations have had the effect of stifling original work in the drug treatment of rare diseases, the position having worsened in recent years.

This trend is illustrated by the history of the introductions of penicillamine and triethylene tetramine for Wilson's disease.
\end{abstract}

IN numbers those who suffer from rare disorders are of course of little consequence. The cost of putting any new medicine on the market is equally prodigious whether it be for a rare or a commonplace ailment, so that it is hardly surprising that no new treatments for the more obscure disorders seem to have emerged for years. At least sixty new preparations appear on the market here each year, nearly all of them being variations of existing medicines for the treatment of diseases which are very prevalent.

If it is churlish to suggest that the system is defective which has brought about the enormous improvements in therapeutics we have witnessed since the 1930s then so be it. I believe that, with respect to the provision of new remedies for rare diseases, things are getting worse rather than better. I shall illustrate this with a short history (Walshe, 1956, 1969, 1973).

Dr J. M. Walshe, having deduced that a metabolite of penicillin might be useful in the rare disorder of metabolism known as Wilson's disease, and having (not without difficulty) persuaded a couple of manufacturers to make some of this material for him, published his first successful results with penicillamine in 1956. Ten years later he was faced with the problem of intolerance to penicillamine by some patients who consequently were in peril of their lives from the disease.

Dr Walshe found a satisfactory substitute to penicillamine in the form of Triethylene Tetramine Dihydrochloride (TRIEN), a laboratory reagent, which however had to be refined for human use. Several patients have now been satisfactorily maintained on this substance for up to 4 years but, incredible though it must appear, Dr Walshe, a practising physician, has still to prepare Trien in his own department, put it into capsules and send it to his patients both here and abroad.

Although the clinical results and methods of preparation have been published in papers such as the Lancet and the Quarterly Journal of Medicine, no pharmaceutical manufacturer has offered to make Trien available and those who have been approached by Dr Walshe have been unable to help. Dr Walshe therefore finds himself fulfilling the role of an apothecary of the time of Jonathan Swift, who alone could do justice to such an absurd situation.

In this probably unique instance, one determined doctor has, at much inconvenience and probably some risk to himself, ensured that his patients shall not be deprived of appropriate therapy. Lesser men would have given up the struggle and I wonder how many good ideas for new approaches to incapacitating but rare disorders have been abandoned because there seems to be no way of realizing them, except on a do-it-yourself basis.

There are two good reasons at least why this general problem should be tackled. One is, as Conan Doyle succinctly put it, that 'singularity is almost invariably a clue'. If we eschew research into treatment of obscure diseases then we are likely to miss useful leads to better treatment of more common disorders. In this context it is perhaps apposite to refer again to penicillamine which, although it was introduced for Wilson's disease, has led to much fruitful study of other and apparently unrelated disorders.

The other reason for our taking the matter seriously is that there is a general duty upon us to do what we can for all patients, and even those with 
obscure but well defined ailments deserve a share of research effort which in part at least should be determined by clinical need.

I have alluded to the economic problem but I feel that this is not in itself an absolute obstacle to the provision of unprofitable medicines to-day any more than it was 20 years ago. A case can be made out for permitting a life-saving drug for a disease which affects only a handful of people to be put on the market without the customary mountain of reports on the compound's pharmacology and toxicology. In fact this is possible here under the terms of a 'Specials' licence. But, despite the existence of this dispensation, it is evident that manufacturers are still reluctant to proceed with preparations such as Trien, and my enquiries into the reason for this lead me to the conclusion that the real block lies in fear of litigation and adverse publicity. There is perhaps an analogy with the feelings of some doctors in the U.S.A. who, fearful of litigation against them, decline to give first-aid at a road accident, or so it is reported.

If this diagnosis is correct and responsible people in the pharmaceutical firms are beginning to lose their nerve, or perhaps feel the game is simply not worth the candle unless the potential market for a new medicine is very large, then the remedy lies in the hands of the nation at large, and especially its doctors, Members of Parliament and journalists. Sir Derrick Dunlop, some time ago, warned everyone that it is not beyond the bounds of possibility that the pharmaceutical goose might become too discouraged to lay any more golden eggs. I suggest that we are beginning to see signs of this. It is idle to hope that the pharmaceutical houses will cease to be objects of public suspicion, for their products possess magical potency for good and ill and, like the whole subject of medicine, readily engage popular attention. However, if there is to be further real progress in drug therapy and especially of the less prevalent maiming diseases, then active, vocal and sustained encouragement of such research inside and outside the commercial sphere is the first essential. I hope that it will be forthcoming.

\section{References}

Doyle, Sir A. Conan The Adventures of Sherlock Holmes. WALSHE, J.M. (1956) Wilson's disease. New oral therapy. Lancet, i, 25.

WALSHE, J.M. (1969) Management of penicillamine nephropathy in Wilson's disease. A new chelating agent. Lancet, ii, 1401.

WALSHE, J.M. (1973) Copper chelation in patients witb Wilson's disease. Quarterly Journal of Medicine, 42, 44K. Z

\section{Discussion}

Mr P. Mould of the National Economic Development Office and member of the Chemicals Economic Development Committee, said that this was a topic which had particularly engaged the attention of the Pharmaceutical Working Party (National Economic Development Office, 1972). Though the importance of the topic was realized, especially in view of the increasing cost of research, no solution was found but it was agreed that the topic should be kept under review. Mr Mould wondered who should be encouraging whom and how. Dr Lyle concluded that only the pharmaceutical industry had the resources to fulfil this need.

\section{Reference}

National Economic Development Office (1972) Focus on Pharmaceuticals. A report by the Pharmaceuticals Working Party of the Chemicals Economic Development Committee. Her Majesty's Stationery Office, London. 